\title{
Free convection between vertical concentric annuli with induced magnetic field when inner cylinder is electrically conducting

\author{
Sarveshanand ${ }^{1}$, Ashok K. Singh ${ }^{2}$ \\ 1 \& 2 Department of Mathematics, Faculty of Science, Banaras Hindu University, Varanasi.
} \\ ${ }^{1}$ DST-Centre for Interdisciplinary Mathematical Sciences, Banaras Hindu University, Varanasi.
}

\begin{abstract}
An analysis is made for the fully developed laminar free convective flow in an open ended vertical concentric annuli with constant heat flux and constant temperature on the inner and outer walls, in the presence of a radial magnetic field. The length of the cylinder is assumed to be infinite and radiation heat transfer from the hot surface is assumed to be negligible. The inner cylinder is taken to be magnetic conducting while the outer cylinder is non-conducting. Buoyancy effect is also considered along with Boussinesq approximation. The induced magnetic field is taken into account arising due to the motion of an electrically conducting fluid. The governing linear simultaneous ordinary differential equations are first obtained in the non dimensional form and solved analytically for the velocity, induced magnetic field, temperature field and then skin-friction and induced current density are obtained. The expressions for the fluid flux and induced current flux in non-dimensional form have been also obtained. The effects of governing physical parameters occurring in the model are shown on the graphs and tables.
\end{abstract}

\section{Keywords}

MHD free convection; Heat flux; Induced magnetic field; Skin-friction; Buoyancy force distribution.

\section{Academic Discipline And Sub-Disciplines}

Fluid Mechanics, Magnetohydrodynamics.

\section{SUBJECT CLASSIFICATION}

Mathematics.

\section{Council for Innovative Research}

Peer Review Research Publishing System

\section{Journal: INTERNATION JOURNAL OF COMPUTERS AND TECHNOLOGY}

Vol. 13, No. 10

editorijctonline@gmail.com

www.ijctonline.com, www.cirworld.com 


\section{INTRODUCTION}

There has been widespread interest in the study of natural convection between two vertical concentric cylinders. In the recent years, the study of transport phenomenon involving the annular geometry has attracted many researchers due to its applicability not only in the field of engineering but also in the field of Geophysics. Some applications of it are the optimization of solidification processes of metals and metal alloys, the study of geothermal sources, the treatment of nuclear fluid debris, the control of underground spreading of chemical wastes and pollutants and the design of magnetohydrodynamic power generators. In 1961, Ramamoorthy [2] has obtained the steady flow of an incompressible fluid between two concentric rotating cylinders and compared the classical hydrodynamic velocity with the magnetohydrodynamic velocity in the presence of a radial magnetic field by neglecting the induced magnetic field. Further, an exact solution for the magnetohydrodynamic flow between two rotating coaxial-cylinders under a radial magnetic field has been obtained by Arora and Gupta [3]. Shaarawi and Sarhan [4] have studied laminar free convective flow in an open ended vertical concentric annuli with rotating inner walls. An investigation for the fully developed free convective flow in vertical annuli with two isothermal boundaries has been obtained by Joshi [5]. Singh et al. [6] have obtained the exact solutions for the fully developed natural convection in open ended vertical concentric annuli with mixed kind of thermal boundary conditions of an electrically conducting fluid under a radial magnetic field. Lee and Kuo [7] have discussed the fully developed, laminar flow in annuli ducts imposed by constant wall temperature at the boundaries by assuming the flow to be Newtonian.

In 2002, Mahmud and Fraser [8] have discussed irreversibility analysis of concentrically rotating annuli in terms of entropy generation in the fluid flow and heat transfer inside cylindrical annuli. An analytical solution through perturbation method and Fourier transform for the natural convection in concentric cylinders with a porous sleeve has been obtained by Leong and Lai [9]. Barletta et al. [10] have discussed the fully developed parallel flow in an annular region filled with a porous medium surrounding a straight cylindrical electric cable. Nada, et al. [11] have investigated heat transfer enhancement in horizontal annuli using nanofluids. Further, Shaija and Narasimhan [12] have investigated the coupled action of conduction, natural convection and surface radiation inside a horizontal annulus numerically.

Most of the above studies on hydromagnetic free convective phenomena involve the cases in which the induced magnetic field is neglected. However, in several physical situations the induced magnetic field has considerable impact and it will be necessary to include its effect in the fluid-dynamical equations. Singh and Singh [13] have investigated effect of induced magnetic field on natural convection in vertical concentric annuli. A study of the time dependent magnetohydrodynamic couette flow in a porous annulus has been made by Jha and Apere [14]. Kumar and Singh [15] have discussed effect of the induced magnetic field on free convection when the concentric cylinders heated/cooled asymmetrically.

In this paper the fully developed laminar free convective flow in open ended vertical concentric annuli has been considered in the presence of a radial magnetic field. The inner cylinder is taken to be magnetic conducting with constant heat flux on it while the outer cylinder is non-conducting and is at a constant temperature. The induced magnetic field is taken into account arising due to the motion of an electrically conducting fluid. The transport equations for the present situation are obtained in the form of simultaneous ordinary differential equations. These equations are first obtained in the non dimensional form and solved analytically for the velocity, induced magnetic field, temperature field, skin-friction and induced current density. The expressions for fluid flux and induced current flux in non-dimensional form have been obtained. The governing equations are also solved for the singular case $(H a=2)$. The effects of governing physical parameters in the model are shown on the graphs and tables.

\section{MATHEMATICAL ANALYSIS}

We have considered here steady laminar fully developed free convective flow of an electrically conducting fluid in the vertical concentric annulus of infinite length. The $z^{\prime}$ - axis is taken as the axis of the co-axial cylinders and $r^{\prime}$ denotes the radial direction measured outward from the axis of the cylinder. The radius of the inner and outer cylinders is taken as $a$ and $b$ respectively. The applied magnetic field of the form $\frac{a B_{0}^{\prime}}{r^{\prime}}$ is taken directed radially outward. The physical model of the considered problem is shown in the figure 1. In the present physical situation, the inner cylinder is taken to be electrical conducting while the outer cylinder as non-conducting. The inner cylinder is at constant heat flux while the outer cylinder is at constant temperature. As the flow is fully developed and cylinder is of infinite length, the variables describing the flow formation depends only on the co-ordinate $r^{\prime}$ and as a result the velocity and magnetic fields are given by $\left(0,0, u^{\prime}\left(r^{\prime}\right)\right)$ and $\left(\frac{a B_{0}^{\prime}}{r^{\prime}}, 0, B_{z^{\prime}}^{\prime}\left(r^{\prime}\right)\right)$ respectively. Thus, under the usual Boussinesq approximation, the basic transport equations for the considered model are obtained as follows:

$$
\begin{aligned}
& \vartheta\left(\frac{d^{2} u^{\prime}}{d r^{\prime 2}}+\frac{1}{r^{\prime}} \frac{d u^{\prime}}{d r^{\prime}}\right)+g \beta\left(T^{\prime}-T_{f}^{\prime}\right)+\frac{a \mu_{e} B_{0}^{\prime}}{\rho r^{\prime}} \frac{d B_{z^{\prime}}^{\prime}}{d r^{\prime}}=0, \\
& \eta\left(\frac{d^{2} B_{z^{\prime}}^{\prime}}{d r^{\prime 2}}+\frac{1}{r^{\prime}} \frac{d B_{z^{\prime}}^{\prime}}{d r^{\prime}}\right)+\frac{a B_{0}^{\prime}}{r^{\prime}} \frac{d u^{\prime}}{d r^{\prime}}=0
\end{aligned}
$$


$\frac{d^{2} T^{\prime}}{d r^{\prime 2}}+\frac{1}{r^{\prime}} \frac{d T^{\prime}}{d r^{\prime}}=0$

The boundary conditions for the velocity, induced magnetic field and temperature field are

$u^{\prime}=0, \frac{d B_{z^{\prime}}^{\prime}}{d r^{\prime}}=0, \frac{d T^{\prime}}{d r^{\prime}}=-\frac{q^{\prime}}{k}, \quad$ at $r=a$,

$u^{\prime}=B_{z^{\prime}}^{\prime}=0, T^{\prime}=T_{f^{\prime}}^{\prime}, \quad$ at $r=b$.

In the above equations, $u^{\prime}$ is the velocity of fluid, $g$ is the acceleration due to gravity, $\mu$ is the coefficient of viscosity, $\mu_{e}$ is the magnetic permeability, $T^{\prime}$ is the temperature of fluid, $T_{f}^{\prime}$ is the ambient temperature, $\vartheta$ is the kinematic viscosity of the fluid and $\beta$ is the coefficient of volume expansion.

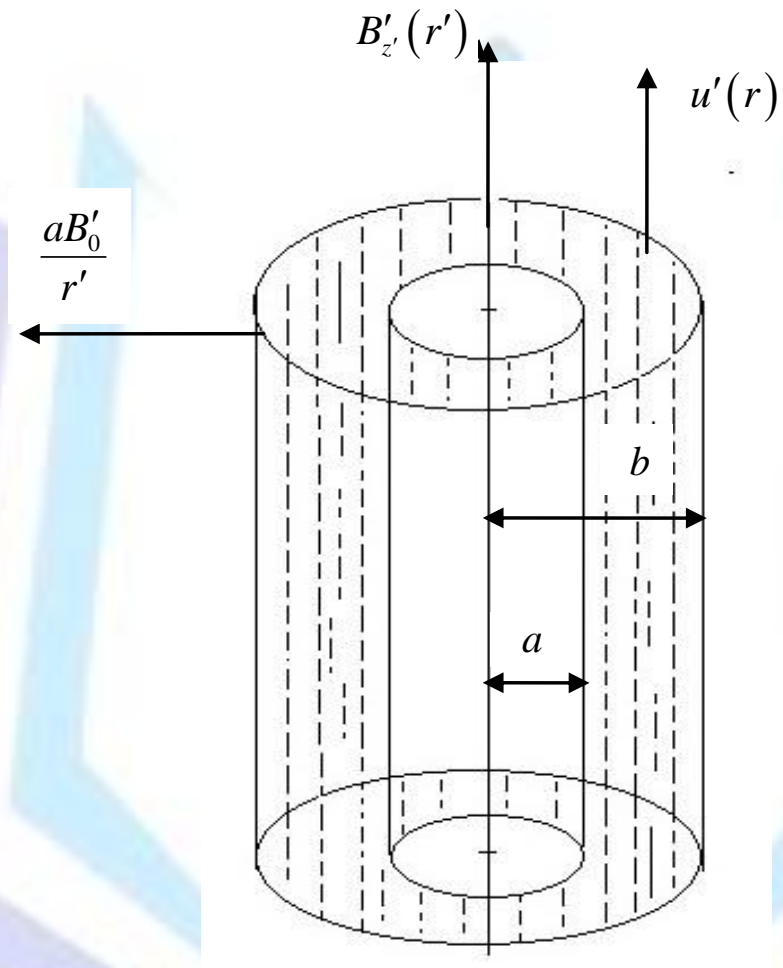

Fig 1: Physical model.

If we introduce the non-dimensional variables as

$u=\frac{u^{\prime}}{U}, r=\frac{r^{\prime}}{a}, \lambda=\frac{b}{a}, T=\left(T^{\prime}-T_{f}^{\prime}\right) \frac{k}{a q}, B=\frac{B_{z^{\prime}}^{\prime}}{a \sigma \mu_{e} B_{0}^{\prime} U}$.

then the governing equations in non-dimensional form are obtained as follows:

$\frac{d^{2} u}{d r^{2}}+\frac{1}{r} \frac{d u}{d r}+\frac{H a^{2}}{r} \frac{d B}{d r}+T=0$

$\frac{d^{2} B}{d r^{2}}+\frac{1}{r} \frac{d B}{d r}+\frac{d u}{d r}=0$

$\frac{d^{2} T}{d r^{2}}+\frac{1}{r} \frac{d T}{d r}=0$ 
The corresponding boundary conditions for the velocity, induced magnetic field and temperature field in dimensionless form are obtained as

$$
\begin{array}{ll}
u=0, \frac{d B}{d r}=0, \frac{d T}{d r}=-1, & \text { at } r=1, \\
u=B=T=0, & \text { at } r=\lambda .
\end{array}
$$

Additional non-dimensional physical parameters appearing in the above equations are the Hartmann number $H a$ and characteristic velocity $U$ of the fluid defined by

$H a=\mu_{e} a B_{0}^{\prime} \sqrt{\sigma / \mu}, U=g \beta a k / \vartheta q$.

The solutions of Eqs. (6)- (8) subject to their appropriate boundary conditions given by Eq. (9), are derived as follows:

$u=D_{1} r^{H a}+D_{2} r^{-H a}+r^{2}\left(D_{4} \log r+D_{5}\right)$,

$B=D_{3}-\frac{1}{H a}\left(D_{1} r^{H a}-D_{2} r^{-H a}\right)+\frac{r^{2}}{4}\left(D_{4}-2 D_{4} \log r-2 D_{5}\right)$;

$T=\log \left(\frac{\lambda}{r}\right)$

where,

$D_{1}=\left(\lambda^{H a} D_{6}-D_{7}\right) /\left(\lambda^{H a}-\lambda^{-H a}\right)$,

$D_{2}=\left(\lambda^{-H a} D_{6}-D_{7}\right) /\left(\lambda^{H a}-\lambda^{-H a}\right)$,

$D_{3}=D_{7}\left(\lambda^{H a}+\lambda^{-H a}\right) /\left(\lambda^{H a}-\lambda^{-H a}\right)-2 D_{6} /\left(\lambda^{H a}-\lambda^{-H a}\right)-D_{8} / H a$,

$D_{4}=1 /\left(4-H a^{2}\right)$,

$D_{5}=-\left\{(\log \lambda+1) /\left(4-H a^{2}\right)+H a^{2} /(4-H a)\right\}$,

$D_{6}=\left\{(\log \lambda+1) /\left(4-H a^{2}\right)+H a^{2} /(4-H a)\right\}$,

$D_{7}=-D_{4} \lambda^{2} \log \lambda-D_{5} \lambda^{2}$,

$D_{8}=\frac{H a \lambda^{2}}{4}\left(D_{4}-2 D_{4} \log \lambda-2 D_{5}\right)$.

The skin-friction in dimensionless form at outer surface of inner cylinder and the inner surface of outer cylinder are obtained from Eq. (11), and are given by

$$
\begin{aligned}
& \tau_{1}=\left(\frac{d u}{d r}\right)_{r=1}=H a\left(D_{1}-D_{2}\right)+2 D_{4}+D_{5}, \\
& \tau_{\lambda}=-\left(\frac{d u}{d r}\right)_{r=\lambda}=H a\left(D_{2} \lambda^{-H a-1}-D_{1} \lambda^{H a-1}\right)-\lambda\left\{D_{4}(1+2 \log \lambda)+D_{5}\right\} .
\end{aligned}
$$

The induced current density along $\theta$-direction obtained from the Maxwell's equation is given by

$J_{\theta}=-\frac{d B}{d r}=D_{1} \lambda^{H a-1}+D_{2} \lambda^{-H a-1}-\frac{r}{2}\left(D_{4}-2 D_{4} \log r-2 D_{5}\right)+\frac{D_{4} r}{2}$. 
The mass flux and the induced current flux of the fluid through the annuli are given by -

$$
\begin{aligned}
& Q=2 \pi \int_{1}^{\lambda} r u d r \\
& =2 \pi\left[\left(\frac{D_{1}}{H a+2}\right)\left(\lambda^{H a+2}-1\right)+\left(\frac{D_{2}}{2-H a}\right)\left(\lambda^{2-H a}-1\right)+\left(\frac{D_{4}}{16}\right)\left\{4 \lambda^{4}(\log \lambda-1)+1\right\}+\left(\frac{D_{5}}{4}\right)\left(\lambda^{4}-1\right)\right], \\
& J=\int_{1}^{\lambda} J_{\theta} d r \\
& =\left(\frac{1}{H a}\right)\left\{D_{1}\left(\lambda^{H a}-1\right)-D_{2}\left(\lambda^{-H a}-1\right)\right\}+\left(\frac{2 D_{5}-D_{4}}{4}\right)\left(\lambda^{2}-1\right)+\left(\frac{D_{4}}{2}\right) \lambda^{2} \log \lambda .
\end{aligned}
$$

\section{Solution for $H a=2$.}

The expressions (11) and (12) for the velocity and induced magnetic field contain the term due to which there is a singularity at $H a=2$. To resolve the singularity at $H a=2$, we have obtained expressions for the velocity, induced magnetic field, skin-frictions, induced current density, fluid flux and induced current flux by solving the govering equations separately for this value of Hartmann number and are given as follows:

$$
\begin{aligned}
& u=E_{1} r^{2}+E_{2} r^{-2}+r^{2} \log r\left(E_{4} \log r+E_{5}\right), \\
& B=E_{3}-\frac{1}{2}\left(E_{1} r^{2}-E_{2} r^{-2}\right)+r^{2} \log r\left(E_{6} \log r+E_{7}\right)+E_{8} r^{2}, \\
& \tau_{1}=\left(\frac{d u}{d r}\right)_{r=1}=2\left(E_{2}-E_{1}\right)+E_{5}, \\
& \tau_{\lambda}=-\left(\frac{d u}{d r}\right)_{r=\lambda}=2\left(E_{1} \lambda-E_{2} \lambda^{-3}\right)+2\left(E_{4}+E_{5}\right) \lambda \log \lambda+2 E_{4} \lambda(\log \lambda)^{2}+\lambda E_{5}, \\
& J_{\theta}=E_{2} r^{-3}+E_{1} r-2 r E_{8}-r E_{7}(2 \log r+1)-2 E_{6} r \log r(\log r+1), \\
& Q=2 \pi\left[\frac{E_{1}}{4}\left(\lambda^{4}-1\right)+E_{2} \log \lambda+\frac{E_{4}}{4} \lambda^{4}(\log \lambda)^{2}+\frac{1}{8}\left(2 E_{4}-E_{5}\right) \lambda^{4} \log \lambda+\left(\frac{E_{4}-2 E_{5}}{32}\right)\left(\lambda^{4}-1\right)\right], \\
& J=\left(\frac{E_{1}}{2}-E_{8}\right)\left(\lambda^{2}-1\right)-\frac{E_{2}}{2}\left(\lambda^{-2}-1\right)-E_{3}(\lambda-1)-E_{6} \lambda^{2}(\log \lambda)^{2}-E_{7} \lambda^{2} \log \lambda .
\end{aligned}
$$

where the constant are given by the following expressions,

$$
\begin{aligned}
& E_{1}=-\frac{E_{9}}{\left(\lambda^{-2}-\lambda^{2}\right)}, E_{2}=\frac{E_{9}}{\left(\lambda^{-2}-\lambda^{2}\right)}, \\
& E_{3}=\frac{\left(\lambda^{-2}+\lambda^{2}\right) E_{9}}{2\left(\lambda^{-2}-\lambda^{2}\right)}-\frac{1}{2} E_{10}, E_{6}=-\frac{1}{4} E_{4}, E_{7}=\frac{E_{4}-E_{5}}{2}, \\
& E_{8}=-\frac{E_{4}-E_{5}}{2}, E_{9}=-\lambda^{2} \log \lambda\left(E_{4} \log \lambda+E_{5}\right), \\
& E_{10}=2 \lambda^{2} \log \lambda\left(E_{6} \log \lambda+E_{7}\right)+2 E_{8} \lambda^{2}, E_{4}=-\frac{1}{8}, E_{5}=-\frac{1}{16} .
\end{aligned}
$$




\section{RESULTS AND DISCUSSION}

The outcomes from the numerical computations of analytical solutions are illustrated via figures and tables in order to analyze the behaviour of the physical parameters on the transport processes resulting from natural convection between coaxial cylinders. Figures 2-4 show the velocity profiles for the different values of the parameter $\lambda$. Fig. 2 shows the variation of velocity for various values of the parameter $H a$, at $\lambda=2$. It is clear from the graph that increasing the value of Hartmann number $\mathrm{Ha}$, the velocity of the fluid decreases.

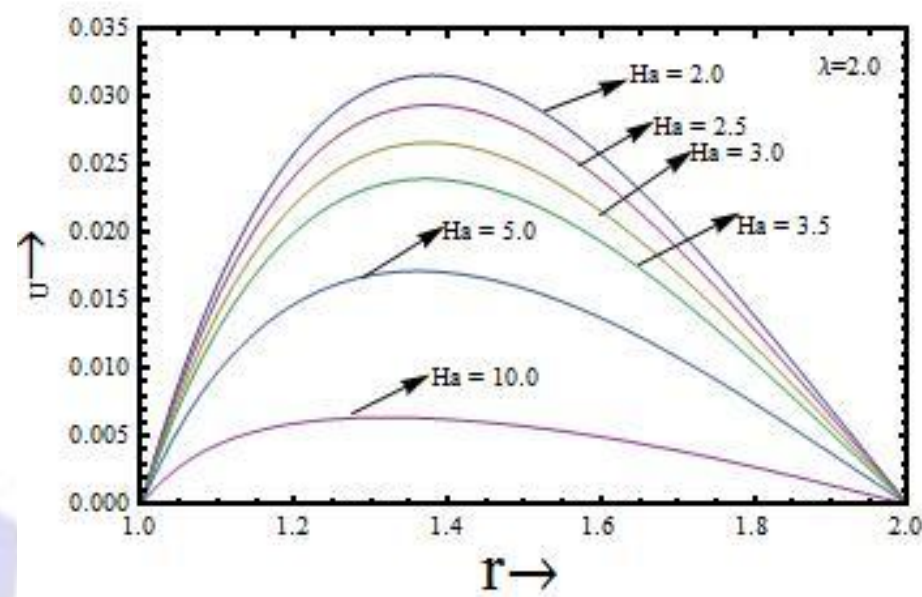

Fig 2: Velocity profile for $\lambda=2.0$

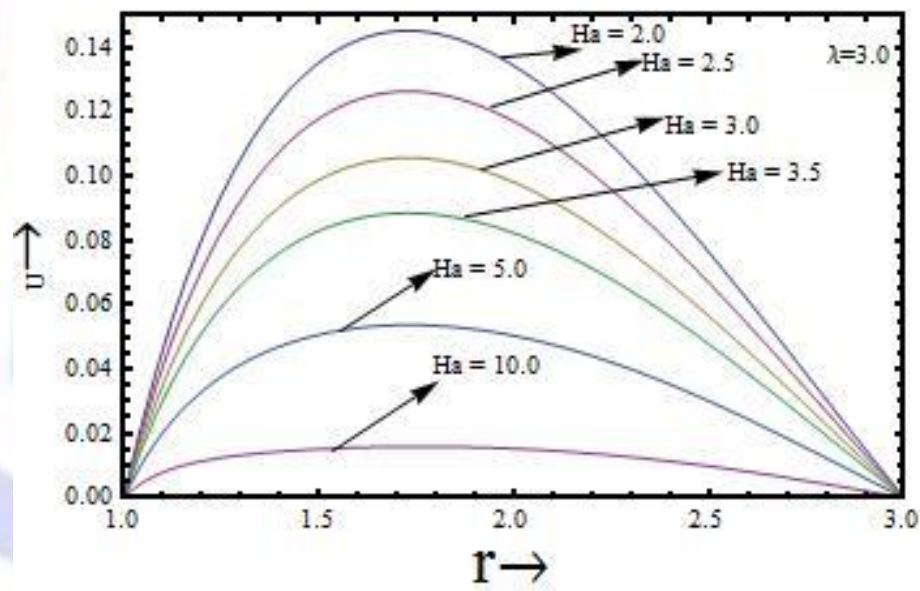

Fig 3: Velocity profile for $\lambda=3.0$

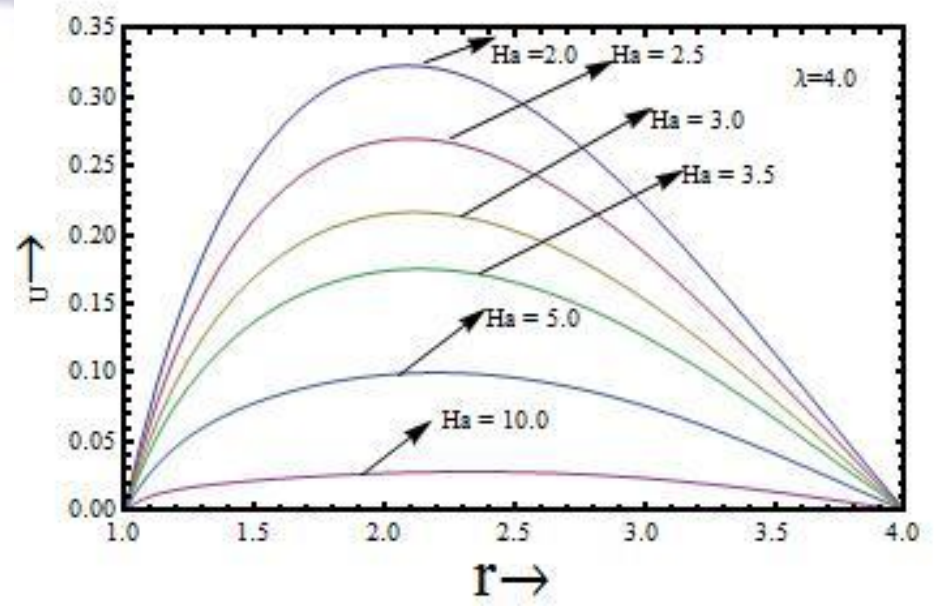

Fig 4: Velocity profile for $\lambda=4.0$ 
Figs. 3 and 4 also show the variation of velocity for various values of the Hartmann number, at $\lambda=3$ and $\lambda=4$ respectively. The graphs clearly show that increasing the value of Hartmann number $H a$, the velocity decreases. From the comparative study of the graphs obtained for the velocity distribution it is clear that as the ratio of outer radius and inner radius increases the velocity increases. The shape of velocity profile has found from parabolic type to flattened type with increase in the value of $\mathrm{Ha}$.

The magnetic field profiles for different values of the parameter $\lambda$ and various values of Hartmann number $H a$, are shown in the Figs. 5-7. Fig. 5 shows the variation of induced magnetic field for various values of Hartmann number at $\lambda=2$. The magnetic field profile shows that increasing the value of Hartmann number $\mathrm{Ha}$, the induced magnetic field decreases near the walls while having same values in the middle of the concentric annulus.

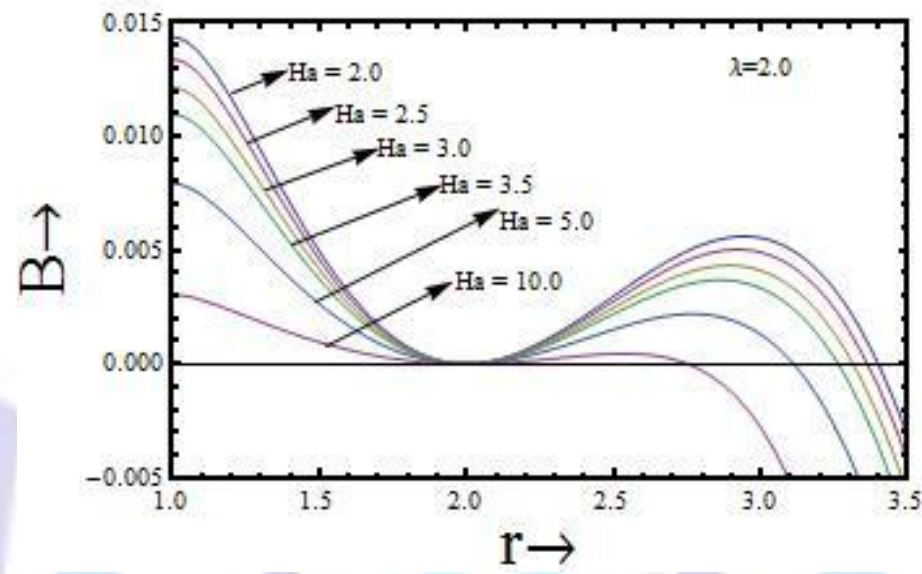

Fig 5: Induced magnetic field profile for $\lambda=2.0$

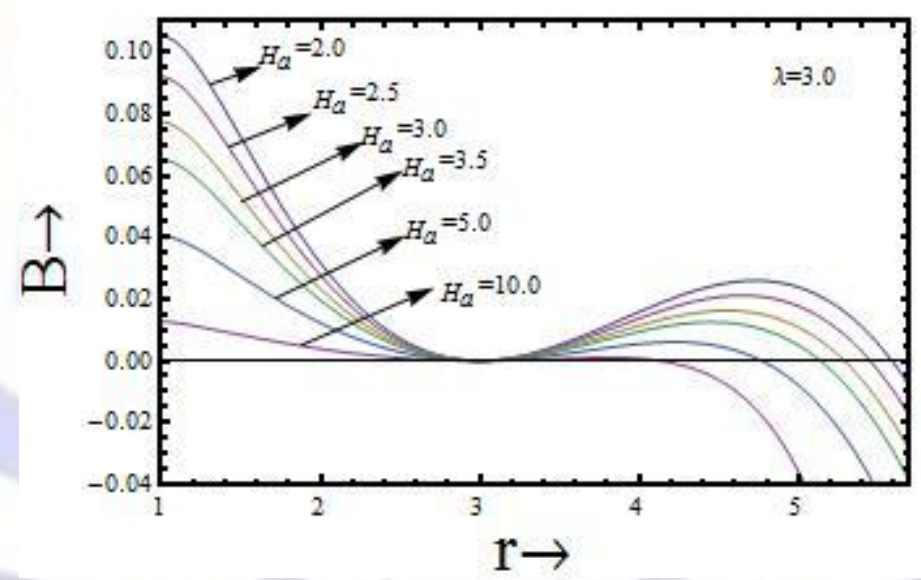

Fig 6: Induced magnetic field profile for $\lambda=3.0$

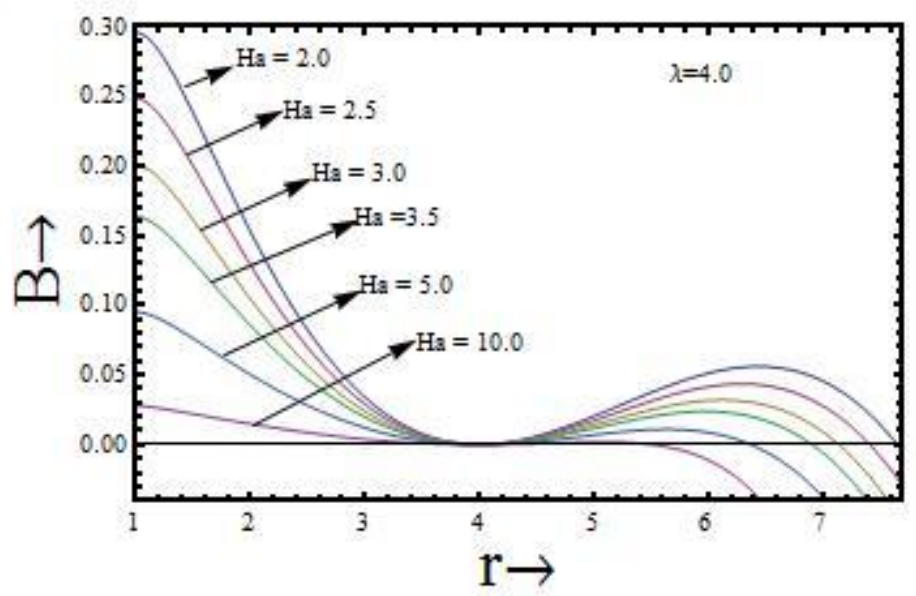

Fig 7 Induced magnetic field profile for $\lambda=4.0$ 
The induced magnetic field profiles at $\lambda=3$ and $\lambda=4$ also shows that the induced magnetic field decreases with increase in the value of Hartmann number. Comparing the graphs of the induced magnetic field profiles given in the Figs. 5,6 and 7 , we find that as the ratio of outer radius to the inner radius increases the induced magnetic field increases.

Figs. 8-10 show the behaviour of induced current density for different values of the parameters $\lambda$ and $H a$.

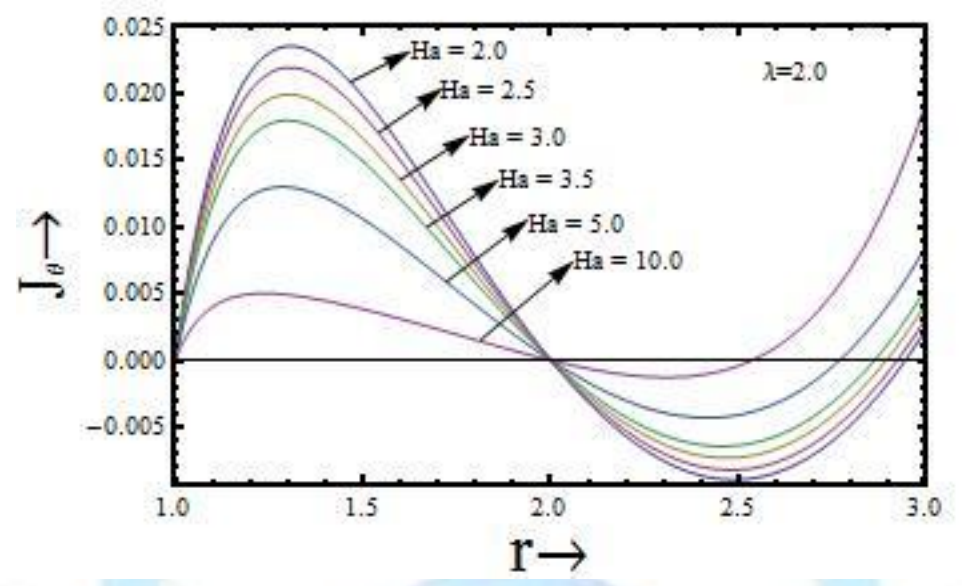

Fig 8:The behaviour of the induced current density for $\lambda=2.0$

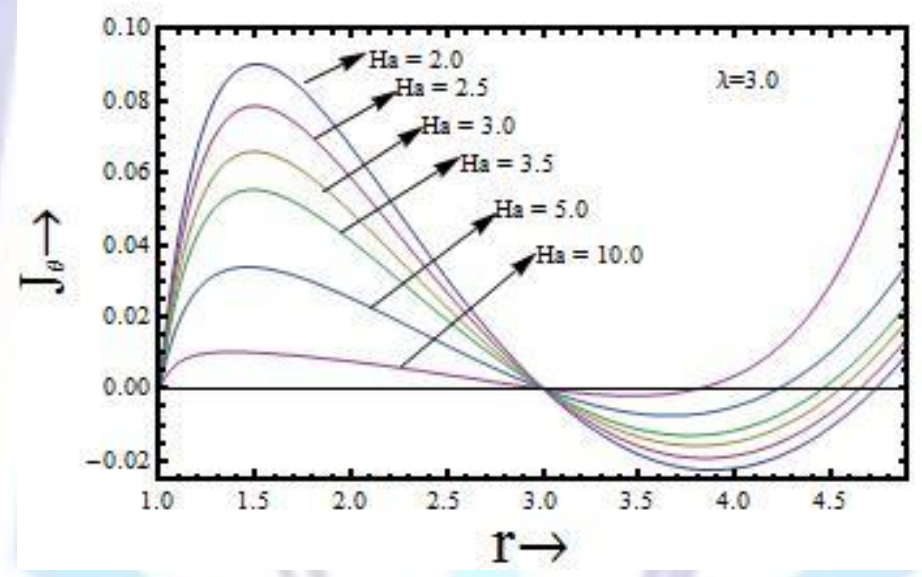

Fig 9:The behaviour of the induced current density for $\lambda=3.0$

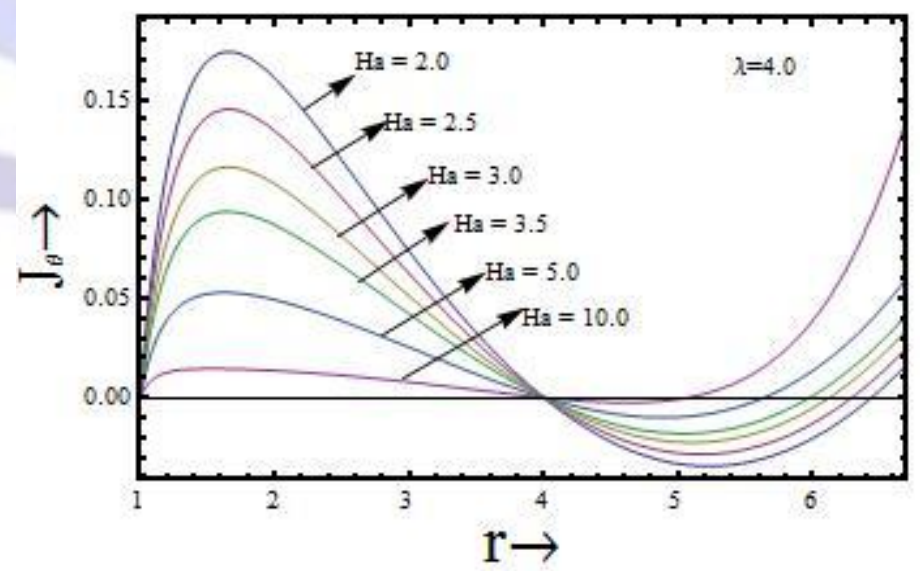

Fig 10:The behaviour of the induced current density for $\lambda=4.0$

The nature of induced current density is found to decrease with increasing the value of $H a$, and it increases with increasing the ratio of outer and inner radii, $\lambda$.

The numerical values of skin-friction at the outer surface of the inner cylinder and at the inner surface of the outer cylinder are given in table 1 . 
Table 1: Numerical values of skin-friction.

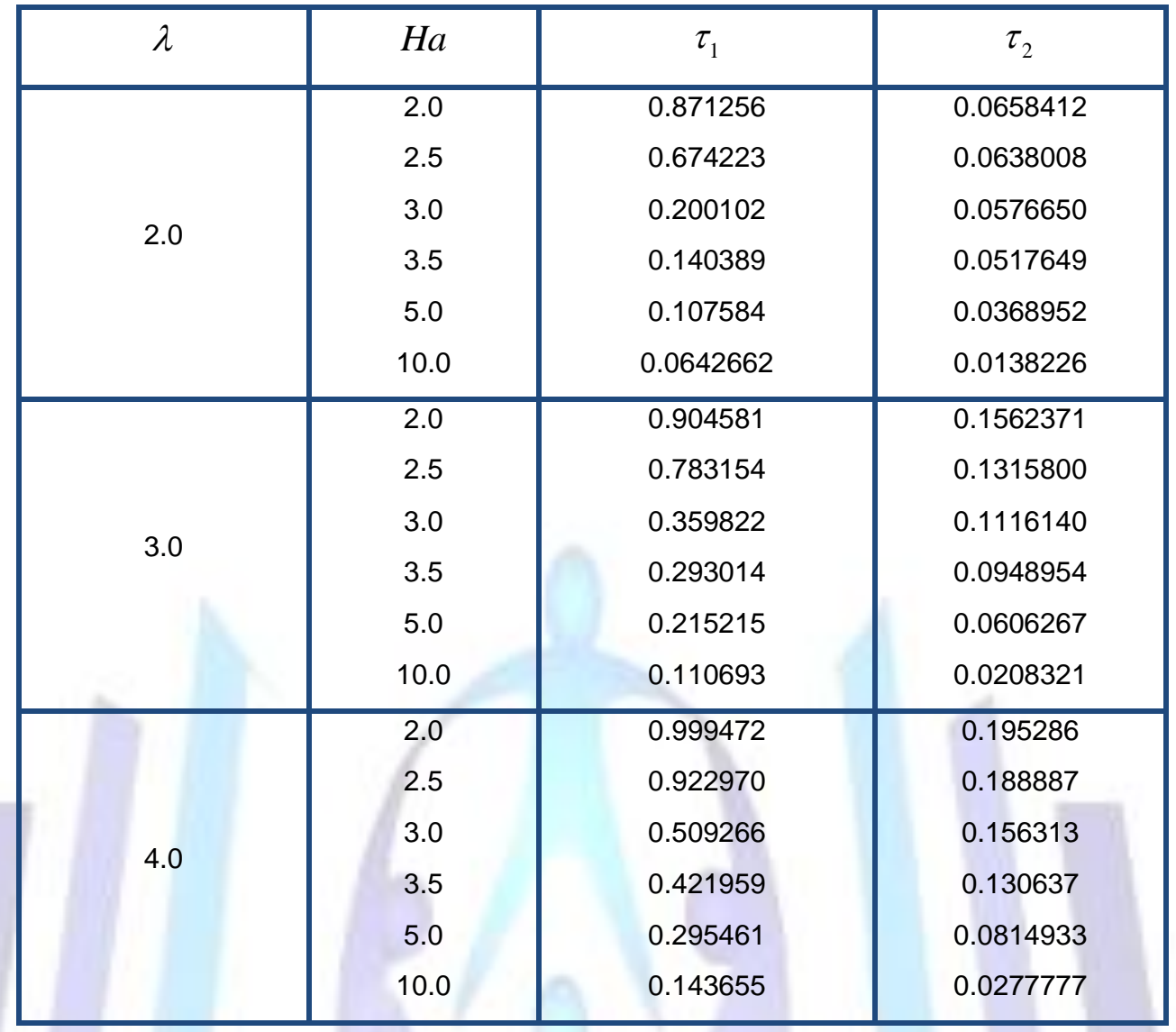

Table 2: Numerical values of fluid flux and induced current flux.

\begin{tabular}{|c|c|c|c|}
\hline$\lambda$ & $H a$ & $Q$ & $J$ \\
\hline \multirow{6}{*}{2.0} & 2.0 & 46.15940 & 0.0143629 \\
\hline & 2.5 & 8.549720 & 0.0133857 \\
\hline & 3.0 & 3.925994 & 0.0121517 \\
\hline & 3.5 & 2.425280 & 0.0109599 \\
\hline & 5.0 & 0.998587 & 0.0079246 \\
\hline & 10.0 & 0.234479 & 0.00304729 \\
\hline \multirow{6}{*}{3.0} & 2.0 & 85.12495 & 0.10249850 \\
\hline & 2.5 & 44.34220 & 0.09194420 \\
\hline & 3.0 & 20.70900 & 0.07736750 \\
\hline & 3.5 & 12.93440 & 0.06519170 \\
\hline & 5.0 & 5.390810 & 0.04041420 \\
\hline & 10.0 & 1.258510 & 0.01266490 \\
\hline \multirow{6}{*}{4.0} & 2.0 & 774.7170 & 0.2957010 \\
\hline & 2.5 & 141.7300 & 0.2483720 \\
\hline & 3.0 & 66.55190 & 0.2005640 \\
\hline & 3.5 & 41.65860 & 0.1635170 \\
\hline & 5.0 & 17.36040 & 0.0951390 \\
\hline & 10.0 & 4.035340 & 0.0278808 \\
\hline
\end{tabular}


It is clear from the table 1 that the ratio of radii of outer to inner cylinders has great impact upon the skin-friction. Increasing the gap between inner and outer cylinders $\tau_{1}$ and $\tau_{2}$ increase. It is also clear from the table 1 that as Hartmann the number $\mathrm{Ha}$ increases, the value of the skin-friction decreases on the outer and inner cylinders.

The numerical values of $Q$ (mass flow rate) and $J$ (induced current flux through the annulus ) at the outer surface of the inner cylinder are given in table 2. As the value of the Hartmann number increases the mass flux of the fluid decreases. A similar behaviour is also obtained in the case of induced current flux. Increasing the value of $H a$, the induced current flux continuously decreases. The gap between two cylinders also affects the mass flux of the fluid and induced current flux. As the gap between the two cylinders increases the mass flux of the fluid and induced current flux both have increasing tendency.

\section{CONCLUSION}

We have analysed the effects of radii ratio parameter $\lambda$ and Hartmann number $\mathrm{Ha}$, on the free convective flow of an electrically conducting fluid generated by imposing constant heat flux on the inner cylinder and constant heat that on the outer when the inner cylinder is conducting while outer is non-conducting in the presence of a radial magnetic field by taking into account the induced magnetic field. It is observed by a comparative study that the effect of the induced magnetic field is to increase the velocity profiles. As the Hartmann number increases, the magnitude of maximum value of the velocity has a decreasing tendency, and the velocity tends to take a uniform value in the middle region for higher values of Hartmann number. The similar flattening tendency is also observed in the induced magnetic field profile and induced current density profile. The magnitude of surface current density is greater on the inner cylinder compared to the outer cylinder. In general, skin-friction, mass flux of fluid and induced current density can be increased by increasing the gap between the cylinders. The effect of magnetic field on the skin-friction can be useful in mechanical engineering for modelling a system. It can be easily obtained a suitable value of Hartmann number for which the value of the skin-friction will be optimum.

\section{Nomenclature}

Radius of inner cylinder $(\mathrm{m})$

$b$

Radius of outer cylinder ( $\mathrm{m}$ )

$g$

Acceleration due to gravity $\left(\mathrm{m} / \mathrm{s}^{2}\right)$

$B_{z^{\prime}}^{\prime}$

Induced magnetic field in $z^{\prime}$-direction $(A / m)$

$B$

Non-dimensional induced magnetic field in $z^{\prime}$-direction $(\mathrm{A} / \mathrm{m})$

$J_{\theta}$

$\mathrm{Ha}$

Induced current density $\left(A / \mathrm{m}^{2}\right)$

$q^{\prime}$

Hartmann number

Heat flux $\left(\mathrm{W} / \mathrm{m}^{2}\right)$

$r^{\prime}, \theta^{\prime}, z^{\prime}$

Cylindrical coordinates $(\mathrm{m}),\left({ }^{(}\right),(\mathrm{m})$

$T_{f}^{\prime} \quad$ Ambient temperature (K)

$T^{\prime} \quad$ Temperature of the fluid (K)

$T$

Temperature of the fluid in non-dimensional form (K)

$u^{\prime}$

Velocity of the fluid along axial direction $(\mathrm{m} / \mathrm{s})$

$u$

Velocity of the fluid along axial direction $(\mathrm{m} / \mathrm{s})$ in non-dimensional form

$U$

Characteristic velocity of the fluid 


\section{Greek symbols}

$\beta$

$\mu_{e}$

$\eta$

$\vartheta$

$\rho$

$\lambda$

$\sigma$
Coefficient of thermal expansion

Magnetic permeability $(H / m)$

Magnetic diffusivity

Kinematic viscosity of the fluid $\left(\mathrm{m}^{2} / \mathrm{s}\right)$

Density of the fluid $\left(\mathrm{kg} / \mathrm{m}^{3}\right)$

Ratio of outer radius and inner radius, b/a

Conductivity of fluid $\left(\mathrm{A}^{2} \mathrm{~S}^{-3} / \mathrm{kgm}^{3}\right)$

\section{Subscript}

1

$\lambda$

$\theta$
Value at inner cylinder

Value at outer cylinder

Along $\theta$-direction

\section{REFERENCES}

[1] Chandrasekhar, S.1961. Hydrodynamic and Hydromagnetic Stability. Oxford, England.

[2] Ramamoorthy, P.1961. Flow between two concentric rotating cylinders with a radial magnetic field. The Physics of Fluids 4,1444-1445.

[3] Arora, K.L., and Gupta, P.R.1971. Magnetohydrodynamic flow between two rotating coaxial cylinders under radial magnetic field. The Physics of Fluids. 15,1146-1148.

[4] El-Shaarawi, M.A.I., and Sarhan, A. 1981. Developing laminar free convection in an open ended vertical annulus with a rotating inner cylinder. Journal of Heat Transfer. 103, 552-558.

[5] Joshi, H.M. 1987. Fully developed natural convection in an isothermal vertical annular duct. International Communications in Heat and Mass Transfer. 14, 657-664.

[6] Singh, S.K., Jha, B.K., and Singh, A.K. 1997. Natural convection in vertical concentric annuli under a radial magnetic field. Heat and Mass Transfer. 32, 399-401.

[7] Lee, Y.M., and Kuo, Y.M. 1998. Laminar flow in annuli ducts with constant wall temperature. International Communications in Heat and Mass Transfer. 25, 227-236.

[8] Mahmud, S., and Fraser, R.A. 2002. Irreversibility analysis of concentrically rotating annuli. International Communications in Heat and Mass Transfer. 29, 697-706.

[9] Leong, J.C., and Lai, F.C. 2006. Natural convection in concentric annulus with a porous sleeve. International Journal of Heat and Mass Transfer. 49, 3016-3027.

[10] Barletta, A., Lazzari, A., Magyari, E., and Pop, I. 2008. Mixed convection with heating effects in a vertical porous annulus with a radially varying magnetic field. International Journal of Heat and Mass Transfer. 51, 5777-5784.

[11] Abu-Nada, E., Masoud, Z., and Hijazi, A. 2008. Natural convection heat transfer enhancement in horizontal concentric annuli using nanofluids. International Communications in Heat and Mass Transfer. 35, 657-665.

[12] Shaija, A., and Narasimham, G.S.V.L. 2009. Effect of surface radiation on conjugate normal convection in a horizontal annulus driven by inner heat generating solid cylinder. International Journal of Heat and Mass Transfer. 52, 57595769.

[13] Singh, R.K., and Singh, A.K. 2012. Effect of induced magnetic field on natural convection in vertical concentric annuli, Acta Mech. Sin. 28(2), 315-323.

[14] Jha, B.K. and Apere, A.C. 2013. Time dependent MHD coquette flow in a porous annulus., Communication in Nonlinear Science and Numerical Simulation.18, 1959-1969.

[15] Kumar, A. and Singh, A. K. 2013. Effect of induced magnetic field on natural convection in vertical concentric annuli heated/cooled asymmetrically. Journal of Applied Fluid Mechanics. 6(1), 15-26. 


\section{Authors' biography with Photo}

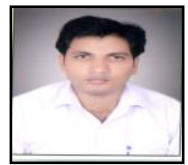

Mr. Sarveshanand has received M. Sc. (Mathematics) degree from Banaras Hindu University in 2009. Currently he is pursuing Ph. D. in the Department of Mathematics, Banaras Hindu University, Varanasi, India. He is also working as a JRF at DST-CIMS, Banaras Hindu University, Varanasi. His research area is Fluid Mechanics and currently working on Magnetohydrodynamic Flows.

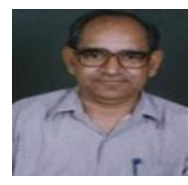

Dr. A. K. Singh is working as a Professor in the Department of Mathematics, Banaras Hindu University, Varanasi, India. In 1979 he earned his Ph.D. in Mathematics from Banaras Hindu University, Varanasi, India. He also earned his D.Sc. in Mathematics from Banaras Hindu University, Varanasi, India, in 1986.

His research interest include Differential Equations, Fluid Dynamics, Numerical Analysis, Magnetohydrodynamic Flows, Flow through porous media, Non-Newtonian fluids, Heat and Mass transfer and Transport processes in cavity. He is currently the president of Mathematical Society Banaras Hindu University, India. He has authored and coauthored over 160 papers in the reputed international and national journals. 